\title{
Joint ESR-EFSUMB recommendation on archiving and reporting ultrasound (US) examinations
}

\author{
Keywords \\ - Ultrasound \\ - Archiving \\ - Information technology systems
}

\begin{abstract}
$\nabla$

The ESR and EFSUMB have issued a joint statement about the necessity of a formal report for all ultrasonographic examinations as well as proper archiving of both reports and images within hospital information technology (IT) systems. Both societies agreed on the importance of availability of US images and reports in clinical practice and agreed on a formal recommendation to their members on this topic.
\end{abstract}

\section{Main messages}

- Availability of reports and images of all US examinations is important in clinical practice as a record of patient status at the time of the studies and as a point of references for future studies, with either US or other imaging modalities

- Both ESR and EFSUMB consider it good clinical practice to provide accurate and retrievable recordings of all ultrasonographic examinations

- Both ESR and EFSUMB recommend to their members to organise workflow in ultrasonography so that proper archiving of both images and reports of all studies can be regularly obtained.

In 2011, the ESR Working Group on Ultrasound (US) conducted a survey on the organisation and practice of US within radiology departments. One of the results of the survey points out that the US studies performed by the laboratories of European hospitals are not always formally reported and that the reports and images of examinations are not always properly archived within the hospital IT systems (1).

Within the framework of the relationships established by the ESR Working Group in US with the European Federation of Societies for Ultrasound in Medicine and Biology (EFSUMB), it has been agreed to prepare and publish a joint recommendation about the necessity of a formal report for all US examinations, as well as of proper archiving of both reports and images.

Archiving of US images and reports is technically feasible, either as a separate archive dedicated solely to US or, better, within the hospital PACS $(2,3)$. It has been recognised that there may be difficulties in providing a network connection when US examinations are performed at the bedside or in an emergency setting, and that large memories may be needed to store large amounts of data when videoclips have to be recorded. However, the widespread use of wireless communication systems and continuous advances in archiving technology should overcome the current problems (4).

From a clinical point of view, availability of US images and reports is recognised by both societies as important in medical practice, as a record of patient status at the time of examination and as a point of reference for future studies. It is believed that compliance with this recommendation will be helpful to improve the quality of services provided to patients.

\section{Recommendation}

$\nabla$

Ultrasonography is a diagnostic technique used in many clinical applications and by different specialists during their practice. US images are not always routinely saved in the hospital PACS systems and, within the same hospital, the reports of the US examinations are often recorded in a variety of archives. However, the availability of US images and reports is important in clinical practice as a record of both in- and out-patients' status at the time of the examination and as a point of reference for future studies, with either US or other imaging modalities. Availability of US images, videos and reports substantially improves the quality of services provided to patients.

Although it is recognised that:

- it may be difficult to find resources to link all US equipment to the IT hospital system and to provide enough memory to store large amounts of data, especially if video clips have to be recorded;

- there may be problems in planning a uniform and complete storage of the examinations' reports, since US can be used in a large variety of ways, from a stand-alone study to a simple and quick guide to a procedure or a physical exam;

both ESR and EFSUMB consider it good clinical practice to provide accurate and retrievable recordings of all US examinations. 
Both societies recommend to their members to organise workflow in US so that proper archiving of images, videos and reports of all studies can be regularly obtained.

\section{Acknowledgements}

$\nabla$

This statement has been kindly prepared by L.E. Derchi (ESR Working Group on Ultrasound Chairman), M. Claudon (ESR Working Group on Ultrasound member), F. Piscaglia (EFSUMB President), N. Gritzmann (EFSUMB Past President) and $\mathrm{T}$. Lorentzen (EFSUMB Publication Committee member) on behalf of the European Federation of Societies for Ultrasound in Medicine and Biology (EFSUMB) and the European Society of Radiology (ESR).
It was approved by the ESR Executive Council, the EFSUMB Executive Bureau, and the EFSUMB Education and Professional Standards Committee in May 2013.

This article was concurrently published in Insights into Imaging (Insights Imaging 2013. DOI: 10.1007/s13244-013-0263-7).

European Society of Radiology (ESR) Neutorgasse 9/2

1010 Vienna

Austria

European Federation of Societies for Ultrasound in Medicine and Biology

(EFSUMB)

28 Portland Place

London W1B 1LS

UK
References:

1 European Society of Radiology. Organisation and practice of radiological ultrasound in Europe: a survey by the ESR Working Group on Ultrasound. Insights Imaging, epub May 29, 2013; DOI: 10.1007/s13244-013-0257-5

2 Hanbidge A, McCallum C, Wilson SR. Introduction of an ultrasound picture archiving and communication system: experience in the first year. Can Assoc Radiol J 1997; 48:162-170

3 Stacul F. Ultrasonography and PACS. Eur J Radiol 1998; 27:S196-S199

4 Dickson BW, Pedersen PC. Wireless image streaming in mobile ultrasound. Telemed JE Health 1998; 16:161-166 